\title{
Resonant microwave transmission from a double layer of subwavelength metal square arrays: Evanescent handedness
}

\author{
C. A. M. Butler, ${ }^{1, *}$ P. A. Hobson, ${ }^{2}$ A. P. Hibbins, ${ }^{1}$ and J. R. Sambles ${ }^{1}$ \\ ${ }^{1}$ Electromagnetic Materials Group, School of Physics, University of Exeter, Stocker Road, Exeter, EX4 4QL, United Kingdom \\ ${ }^{2}$ Smart Technologies Team, QinetiQ Ltd, Cody Technology Park, Ively Road, Farnborough, GU14 OLX, United Kingdom \\ (Received 19 September 2011; revised manuscript received 16 May 2012; published 26 December 2012)
}

\begin{abstract}
A double layer of identical subwavelength metal patch arrays is experimentally shown to be electromagnetically chiral due to the evanescent coupling of the near fields between nonchiral layers-it exhibits "evanescent handedness." Despite each layer being intrinsically isotropic in the plane with four mirror planes orthogonal to the plane of the structure, circular dichroism, leading to significant polarization rotation, is found in the resonant microwave transmission for any incident linear polarization.
\end{abstract}

DOI: 10.1103/PhysRevB.86.241109

PACS number(s): 81.05.Xj, 41.20.Jb, 78.20.Ek, 78.67.Pt

Chirality is determined by the symmetry of a structure. The most obvious example of chirality is the symmetry of our hands. The left and right may be identical to their mirror image, however, one cannot be translated to become the other. Structures that are electromagnetically chiral give rise to selective reflection or transmission of one specific circular polarization state of electromagnetic (EM) radiation, and may induce rotation of the plane of linearly polarized radiation on transmission or reflection. Such responses arise in a variety of ways. Sugar solution, for example, gives rise to rather weak rotations of polarization due to the intrinsic chirality of the sugar molecules. ${ }^{1}$ Anisotropic (uniaxial) liquid crystals, when arranged so that the "director" of their molecules twists slowly (compared to the wavelength of incident radiation) with distance perpendicularly away from the surface, may cause very strong polarization rotation. This phenomenon is used extensively in flat screen liquid crystal displays (LCDs). ${ }^{2}$

Recently, the concept of chirality in subwavelengthstructured materials ("metamaterials" see Refs. 3 and 4) has created much interest in the EM research community. Investigations into the induced optical activity have explored arrays of both chiral and nonchiral elements confined to a plane. These have included studies of optical activity in intrinsically nonchiral but anisotropic metamaterials, ${ }^{5}$ asymmetric transmission of EM radiation through anisotropic planar chiral structures, ${ }^{6}$ and both planar and nonplanar chiral metamaterials for creating a negative refractive index. ${ }^{7,8}$ Researchers have also considered other structures with relatively complex chiral elements including multiple layers of rosettes ${ }^{9}$ and gammadions, ${ }^{10,11}$ as well as much simpler geometries such as wire pairs ${ }^{12,13}$ and rotated crosses. ${ }^{14,15}$ In addition, a new aspect of chirality has recently been presented by Plum et al., ${ }^{16}$ showing that asymmetric transmission of circularly polarized waves can be achieved through any lossy periodically structured system for oblique incidence.

The work presented in this rapid communication provides a fresh development in this field. We demonstrate chirality at normal incidence arising from two closely spaced isotropic layers with four mirror planes formed of nonchiral elements. Each layer is composed of identical square arrays of subwavelength square metallic patches. Each array is nondiffracting, and arranged with its sides parallel to the axes of the square lattice. The two layers are parallel spaced such that, at frequencies below the onset of diffraction, they are situated within a decay length of each other's evanescent fields, with the axes of one array rotated relative to the other. Note that such samples should be carefully differentiated from arrays of split rings, cut wire pairs, or rotated crosses, etc., in which each element is rotated so that its mirror plane does not coincide with a mirror plane of the array, and in each layer elements are rotated differently.

Metal patch arrays are well documented as finding applications as low pass filters, frequency selective surfaces, and antenna arrays. ${ }^{17}$ There also exists an extensive body of work exploring double layer patch arrays. ${ }^{17,18}$ For two aligned and unrotated patch arrays waveguidelike resonant modes in the cavity formed between the patch layers are excited. ${ }^{19}$ In isolation, or when the separation between the patch arrays is large compared with the wavelength, these arrays exhibit an isotropic EM response, and can be satisfactorily represented using a simple effective medium approach. This is evidenced by the fact that the normal incidence EM response of a single array is not dependent on the angle between the plane of incidence and the axes of the lattice (azimuth $\varphi$ ). Furthermore, as reported by some of the present authors, ${ }^{20}$ the EM response of a dual layer system, spaced by approximately a wavelength, is independent of translational displacement in the plane of one array with respect to the other. However Ref. 20 also demonstrates that when the layer spacing between the two patch arrays is smaller, the EM fields associated with evanescent diffracted orders overlap and translational misalignment of the structure changes the response. Now consider a closely spaced, double layer of metal patches, in which the arrays are rotated by an angle (other than $45^{\circ}$ or $90^{\circ}$ ) with respect to each other; this is the geometry of interest in the present work (Fig. 1). Provided the two layers are not in intimate electrical contact, which would form a nonchiral structure, then the system is locally "handed" and polarization rotation may occur. Since each patch array has a subwavelength periodicity, it effectively behaves as an isotropic in-plane layer whose EM response (and polarization rotation) is independent of the azimuth angle of incidence $(\varphi)$ and therefore the incident linear polarization.

By measuring both the phase and amplitude of the transmitted signal using a linearly polarized detector at appropriate output polarization angles, the ellipticity induced by the 


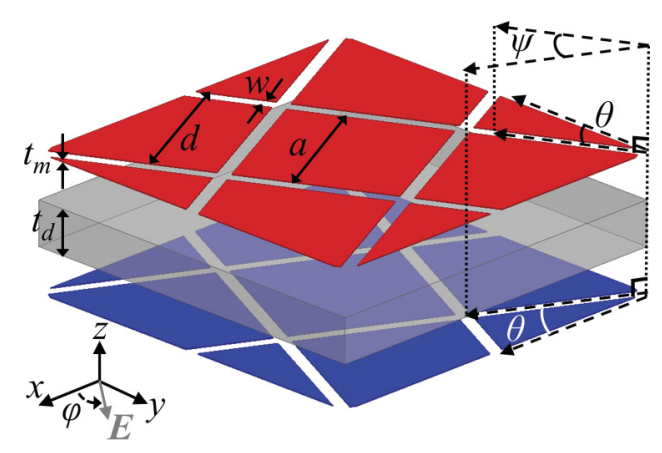

FIG. 1. (Color online) Exploded view of the modeled commensurate unit cell for the rotated patch array structure, where $w=0.3 \mathrm{~mm}, a=3.5 \mathrm{~mm}$, and $d=3.8 \mathrm{~mm}, t_{m}=17.5 \mathrm{um}$, $t_{d}=0.508 \mathrm{~mm}, \psi=36.88^{\circ}$, and $\theta=26.56^{\circ}$. Azimuthal angle $\varphi$ for the detected electric-field vector polarization (parallel to the $+x$ axis $\varphi=0^{\circ}$ ).

structure is deduced, and the circular dichroism quantified. The key observation from these findings is that while neither the array as a whole, nor its constituent elements are handed, the evanescently coupled combination gives a strong chiral response.

The experimental sample (Fig. 1) consists of two arrays of copper patches $17.5 \mu \mathrm{m}$ thick separated by $0.508 \mathrm{~mm}$ of dielectric (Rogers RO $4003 \mathrm{C}$ ). The patch arrays are rotated by $\psi=36.88^{\circ}$ with respect to each other and $\theta= \pm 26.56^{\circ}$ to the $x$ or $y$ axis. The angle $\theta$ is equivalent to a $(2,1)$ rotation of each array in opposite directions. It is chosen to give a commensurate geometry defined by the smallest possible double-layer unit cell. This unit cell is $\sqrt{5}$ larger than that of an unrotated double layer system, and therefore leads to a reduction of the frequency at which diffraction first occurs by $\sqrt{5}$. This commensurate geometry is chosen to allow the EM response of this computationally expensive structure to be modeled $^{21}$ using a unit cell and periodic boundary conditions in the $x y$ plane.

Linearly polarized microwave radiation (electric vector parallel to the $x$ direction, $\varphi=0^{\circ}$ shown in Fig. 1) is emitted from a rectangular waveguide horn antenna. This is placed at the focus of a collimating mirror to give plane waves incident normally on the sample. The transmitted beam is collected in a similar manner via a second mirror having a rectangular waveguide horn detector antenna at its focus. This detecting antenna can be azimuthally rotated to allow quantification of any linear polarized output component. In what follows the polarization state of each horn is defined by the angle $(\varphi)$ the electric-field vector makes with the $x$ axis (shown in Fig. 1).

A set of modified patch resonances are evident as two peaks in the polarization conserving transmission response (Fig. 2 inset, 21.8 and $23.4 \mathrm{GHz}$ ). These modes are not the simple patch array modes that occur if the arrays are aligned (not rotated) ${ }^{17}$ but are combination resonances. Within the data are extra features that are apparent as two transmission peaks in the polarization conversion signal (Fig. 2). There are two strong peaks at 18.21 and $25.08 \mathrm{GHz}$, with a third much weaker feature at $23.88 \mathrm{GHz}$. The continuous line on

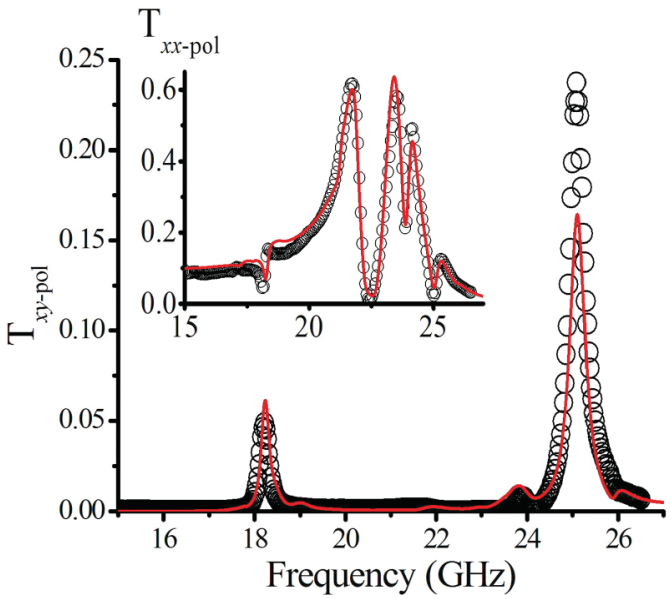

FIG. 2. (Color online) Experimentally observed (circles) and numerically modeled (line) EM response recorded at normal incidence for polarization conversion $\left(T_{x y \text {-pol }}\right)$. Inset: Experimentally observed signal (circles) for polarization conserving transmission $\left(T_{x x \text {-pol }}\right)$. Subscripts refer to the orientation of the electric field vector in the incident and detected beams, respectively.

the figure is the best-fit using FEM modeling. ${ }^{21}$ Parameters fitted were the frequency dependent permittivity $(3.55 \pm 0.05$ over the experimental frequency range), dielectric loss tangent $(\tan \delta=0.008)$, and the $x y$ offset of the layers $(x=0.08$ $\mathrm{mm}, y=0.06 \mathrm{~mm}$ ). Note that such further modeling (not shown) demonstrates that this offset is responsible for the weak feature arising at $23.88 \mathrm{GHz}$ (i.e., the center of rotation of one array is not exactly in the center of the unit cell of the other).

The two dominant peaks (at 18.21 and $25.08 \mathrm{GHz}$ ) arise from coupling of the evanescent diffracted fields within the dielectric between the layers to establish resonant modes. The polarization converting modes rely upon the structure of each subwavelength array decomposing the incident radiation into the two orthogonal components associated with the square geometry. The subwavelength nature dictates that the transmitted radiation is evanescent, crucially containing near field non-diffraction-limited information regarding the subwavelength sample geometry and orientation. The coupling of the evanescent diffracted field from each array forms the chiral resonant mode that gives the rotation of linear polarization. Of course, a polarization converting response implies that the structure could be considered simply as a birefringent material. However, symmetry dictates, and experiment confirms, that the sample may be rotated by any azimuth angle, or equally illuminated by any azimuth angle of linear polarization, yielding the same polarization conversion response.

The resonant modes that induce strong polarization rotation (with respect to the distance between metal patches) have chiral EM field profiles within the dielectric region. This chirality is illustrated in Fig. 3, where the electric field vectors within the commensurate unit cell are shown for $18.21 \mathrm{GHz}$ at three planes at different heights in the $z$ direction (propagation direction). These vectors clearly show the azimuthal rotation of the fields within the layer. Simultaneously, a small proportion 

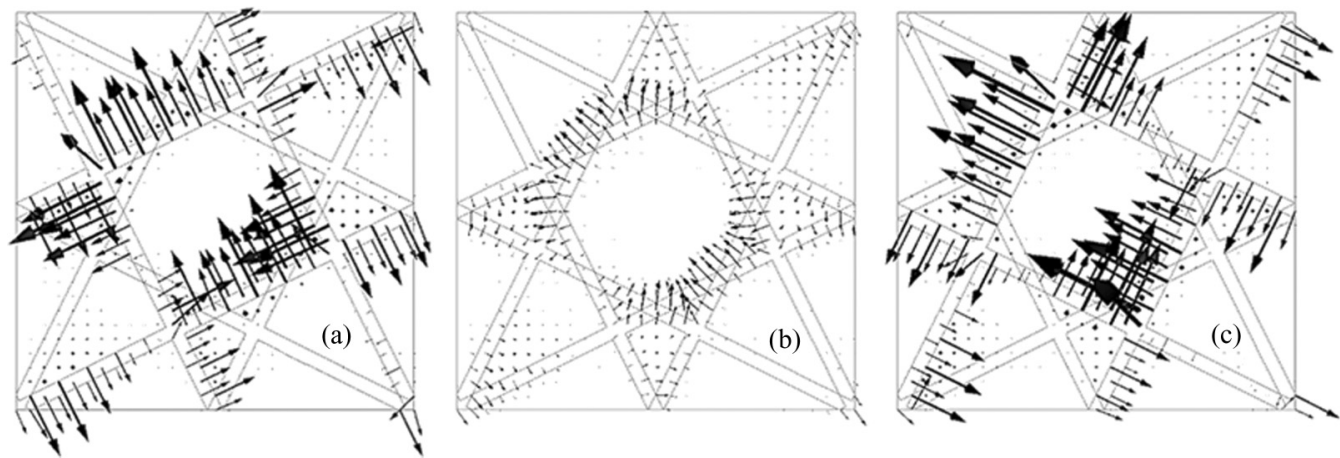

FIG. 3. Vector electric field profiles for $18.21 \mathrm{GHz}$, shown for a unit cell in the $x y$ plane for (a) $z=0.254 \mathrm{~mm}$, on the upper patch dielectric boundary; (b) $z=0 \mathrm{~mm}$, at the center of the dielectric, and (c) $z=-0.254 \mathrm{~mm}$, at the lower patch dielectric boundary. Electric field enhancement varies from 0 to 2000 .

(evident as the nonzero polarization conserving transmission in Fig. 2 inset) is transmitted unrotated at this frequency. So, the resultant EM wave is elliptically polarized. This ellipticity is readily quantified by rotating the detector (which is polarization selective) and measuring the transmitted signal as a function of that rotation $(\varphi)$. These results are illustrated in Fig. 4(a) for modes at 18.21 and $25.08 \mathrm{GHz}$. The degree of ellipticity is shown by the extent to which these "figure-ofeights" do not quite close to a zero crossing. Figure 4(b) shows how the output polarization rotates through $180^{\circ}$ as one passes through the resonance.

From the linear polarization conserved and converted data (amplitude and phase measurements), the circularly polarized response of the double layer can be deduced. If the linearly polarized input wave comprises of equal amounts of right and left circular polarized components (RHCP and LHCP, respectively), then the circular dichroism is given by Ref. 4 :

$$
C D=\frac{A_{R}^{2}-A_{L}^{2}}{A_{R}^{2}+A_{L}^{2}}=\frac{-2 E_{x} E_{y} \sin \varphi_{x y}}{E_{x}^{2}+E_{y}^{2}} .
$$

Here $A_{R}$ and $A_{L}$ are the RHCP and LHCP complex amplitudes of the transmitted signal, respectively, $E_{x}$ and $E_{y}$ are the co-polarized and cross-polarized transmission amplitude, and $\varphi_{x y}$ is the difference in phase between the co-polarized and cross-polarized transmission. Results shown in Fig. 5 illustrate the left handed circularly polarized nature of the resonances occurring at 18.21 and $25.08 \mathrm{GHz}$, implying that the structure has a strong chiral response at these resonant frequencies. To give further confirmation of this, using the idealized perfect conductor model as shown in Fig. 1, the chirality parameter $(\kappa)$ has been evaluated ${ }^{4}$ and, as shown in Fig. 5 inset, has two strong resonant peaks as expected.

This simple structure provides a fresh paradigm for chiral electromagnetic metamaterials - where evanescent fields couple rotated isotropic layers of high symmetry to give handedness. When the two square-symmetric subwavelength patch arrays are several wavelengths apart, there is no polarization rotation as both arrays, for normal incidence radiation, have electromagnetic rotational invariance due to their square symmetry. However, as they are moved closer together, to within less than a wavelength of the radiation, strong optical activity (polarization rotation) occurs. This ability to rotate the plane of polarization is independent of azimuth angle but is frequency selective, depending on the form of the patches one chooses and the array symmetry. Hexagonal arrays with triangular patches, which individually
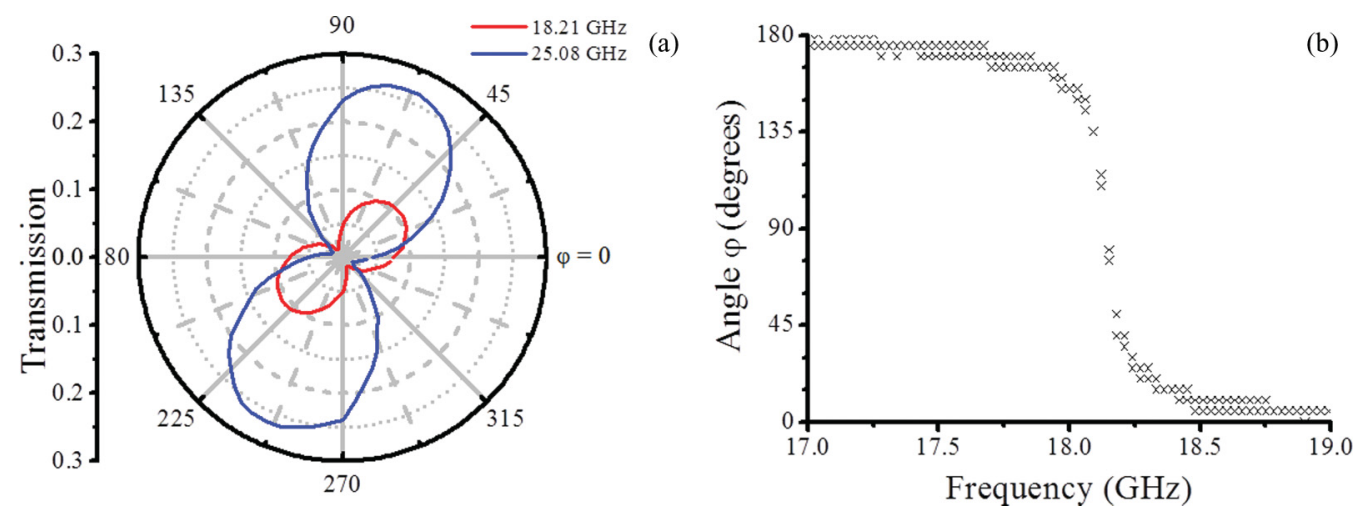

FIG. 4. (Color online) (a) Polar plot presenting the experimental transmission as a function of detector azimuth angle $\varphi$ for combination resonant modes at 18.21 and $25.08 \mathrm{GHz}$. (b) Experimental detector azimuth angle $\varphi$ at which maximum transmission occurs as a function of frequency. 


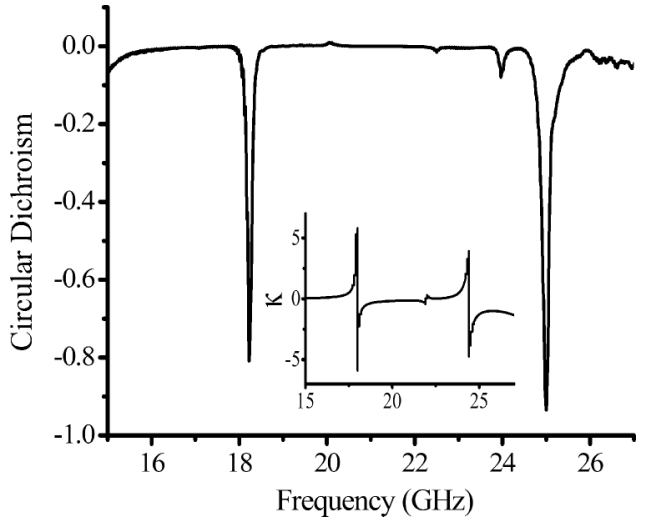

FIG. 5. Circular dichroism as a function of frequency calculated using the experimentally recorded complex linearly co-polarized and cross-polarized transmission results from Fig. 2. Inset: Chirality parameter $(\kappa)$ for the idealized structure as shown in Fig. 1. are nonchiral, will show similar behavior but at different frequencies. This structure has been explored here in the microwave regime, however, it could be scaled down in physical size to operate in the $\mathrm{THz}$, and even the optical regime. Care would need to be taken to ensure an array of near PEC metal patches, possibly leading to thicker metallic layers to avoid losses. Furthermore, although presently not amenable to modeling, a sequence of the arrays with each rotated by a small amount relative to the other will act as an adiabatic polarization rotator although how much radiation would transmit through such a structure is presently unknown.

The authors wish to acknowledge the financial support of QinetiQ and the EPSRC (UK) for funding CAMB through the Industrial CASE scheme (No. 08000346). The authors also wish to acknowledge the help of A. C. Oxlade for assisting in the collection of data. *celia.butler@ex.ac.uk

${ }^{1}$ F. J. D. Arago, Mémoires De La Classe Des Sciences Math. Et Phys. De l'Institut Impérial De France 1, 93 (1811).

${ }^{2}$ P. Yeh, and C. Gu, Optics of Liquid Crystal Displays, 2nd ed. (Wiley-Blackwell, London, 2010), p. 770.

${ }^{3}$ D. R. Smith, J. B. Pendry, and M. C. K. Wiltshire, Science 305, 788 (2004).

${ }^{4}$ B. Wang, J. Zhou, T. Koschny, M. Kafesaki, and C. M. Soukoulis, J. Opt. A. 11, 114003 (2009).

${ }^{5}$ E. Plum, V. A. Fedotov, and N. I. Zheludev, Appl. Phys. Lett. 93, 191911 (2008).

${ }^{6}$ V. A. Fedotov, P. L. Mladyonov, S. L. Prosvirnin, A. V. Rogacheva, Y. Chen, and N. I. Zheludev, Phys. Rev. Lett. 97, 167401 (2006).

${ }^{7}$ B. Wang, J. Zhou, T. Koschny, and C. M. Soukoulis, Appl. Phys. Lett. 94, 151112 (2009).

${ }^{8}$ Z. Li, R. Zhao, T. Koschny, M. Kafesaki, K. B. Alici, E. Colak, H. Caglayan, E. Ozbay, and C. M. Soukoulis, Appl. Phys. Lett. 97, 081901 (2010).

${ }^{9}$ E. Plum, J. Zhou, J. Dong, V. A. Fedotov, T. Koschny, C. M. Soukoulis, and N. I. Zheludev, Phys. Rev. B 79, 035407 (2009).
${ }^{10}$ K. Konishi, M. Nomura, N. Kumagai, S. Iwamoto, Y. Arakawa, and M. Kuwata-Gonokami, Phys. Rev. Lett. 106, 057402 (2011).

${ }^{11}$ E. Hendry, T. Carpy, J. Johnston, M. Popland, R. V. Mikhaylovskiy, A. J. Lapthorn, S. M. Kelly, L. D. Barron, N. Gagegaard, and M. Kadodwala, Nat. Nanotechnol. 5, 783 (2010).

${ }^{12}$ Y. Ye and S. He, Appl. Phys. Lett. 96, 203501 (2010).

${ }^{13}$ Z. Wu, J. Zhu, M. Jia, H. Lu, and B. Zheng, Microwave Opt. Technol. Lett. 53, 163 (2011).

${ }^{14}$ J. Zhou, J. Dong, B. Wang, T. Koschny, M. Kafesaki, and C. M. Soukoulis, Phys. Rev. B 79, 121104 (2009).

${ }^{15}$ Z. Li, H. Caglayan, E. Colak, J. Zhou, C. M. Soukoulis, and E. Ozbay, Opt. Express 18, 5375 (2010).

${ }^{16}$ E. Plum, V. A. Fedotov, and N. I. Zheludev, J. Opt. 13, 024006 (2011).

${ }^{17}$ B. A. Munk, Frequency Selective Surfaces: Theory and Design (Wiley-Interscience, New York, 2000), p. 416.

${ }^{18}$ T. K. Wu, and C. H. Chan, Frequency Selective Surfaces and Grid Array (Wiley-Interscience, New York, 1995), Chaps. 1 and 2.

${ }^{19}$ A. P. Hibbins, J. R. Sambles, C. R. Lawrence, and J. R. Brown, Phys. Rev. Lett. 92, 143904 (2004).

${ }^{20}$ M. C. Taylor, A. P. Hibbins, and J. R. Sambles, Phys. Rev. B 86, 035126 (2012).

${ }^{21}$ HFSS, Ansoft Corporation, Pittsburgh, PA, USA. 